\title{
Slowly Variable Early F-type Stars
}

\section{L.A. Balona}

South African Astronomical Observatory, P.O. Box 9, Observatory 7935, Cape, South Africa

\section{K. Krisciunas}

\author{
Joint Astronomy Centre 660 N. A'ohōkū Place, University Park, Hilo, \\ Hawaii 96720, USA
}

\begin{abstract}
A third periodicity is found in the early F-type star $\gamma$ Dor which probably rules out an interpretation in terms of differential rotation of a spotted star. One of the two periods in 9 Aur is not visible in the radial velocity. This is not explained by the star spot model, but may indicate that the two periodicities are nonradial pulsations of different spherical harmonic degrees.
\end{abstract}

\section{Introduction}

In recent years there has been growing evidence for the existence of a new class of variable among main-sequence early F-type stars. These stars show periodic light variations of many hours - too long to be attributed to $p$-mode pulsations characteristic of $\delta$ Scuti stars. They are all early-F dwarfs on, or beyond, the red edge of the $\delta$ Scuti instability strip. Few of these stars have been well studied, but whenever sufficient observations are available, they are found to be multiperiodic.

\section{Observations}

By far the best studied member of this class is $\gamma$ Dor, an F1V star in which two sinusoidal variations with periods $P_{1}=0.7570$ and $P_{2}=0.7334 \mathrm{~d}$ are present (Balona, Krisciunas \& Cousins 1994). For two weeks in 1994, November, we obtained photometry of $\gamma$ Dor from SAAO (LAB) and CTIO (KK). Observations from two sites are required to ensure that $P_{1}$ and $P_{2}$ in $\gamma$ Dor are separate periodicities. The latest observations eliminate the possibility that only one period is present. Balona et al., (1994) found marginal evidence for a third periodicity at $P_{3}=0.678 \mathrm{~d}$, not too different from the well known periods at $P_{1}$ and $P_{2}$ d. Our most recent data again show the presence of $P_{3}$ and we conclude that is most probably real. This component was not present before 1993 .

Krisciunas et al. (1995) provide significant evidence that the photometric variations of 9 Aur are due to nonradial $g$-mode pulsations of low spherical harmonic order. Their radial velocity data, obtained by $R$. Griffin with CORAVEL 
at Haute Provence, exhibit variations entirely consistent with only the longer of the two periods of 9 Aur. The preliminary conclusion is that $P_{2}$ comes from an $\ell=1$ or 2 spherical harmonic, while $P_{1}$ comes from a higher order harmonic.

A multi-longitude campaign on 9 Aur was carried out in 1994 December and 1995 January. These observations show 9 Aur exhibiting the largest photometric amplitudes seen to date. It ranged in brightness by 0.090 in the $V$-band, and by 0.124 in the $B$-band. As with $\gamma$ Dor, the $B-V$ colours of 9 Aur phase up with the $V$-band light curve in the sense that the bluest colour (i.e., hottest temperature) occurs when the star is brightest.

\section{Star spots or nonradial pulsations?}

There are only two viable hypotheses to explain the light variations in these stars: star spots or nonradial pulsations. Since they are multiperiodic, the star spot model presumes differential rotation. In order to understand the rather large frequency differences, Mantegazza, Poretti \& Zerbi (1994) assume that the true periods are actually harmonics and/or aliases of the observed periods. Since there is little or no observed power at the fundamental frequencies, the diametrically opposed spots must be on the same latitude and must have equal sizes.

In the case of $\gamma$ Dor the situation is simple, since the periods are close enough to be understood in terms of differential rotation. However, the detection of a third period implies an implausibly large differential rotation. In the case of 9 Aur, it is difficult to understand why one of the periods is not visible in the radial velocity observations.

In a study of $\gamma$ Dor, Balona et al. (1994) found that spots would have to be larger than $20^{\circ}$ in radius and more or less on the same latitude. The spots then interact at every beat period (about $23.5 \mathrm{~d}$ ), in contradiction to the fact that the two principal periods have been extremely stable over the last 12 years with little or no change in amplitude.

In our opinion, there seems little doubt that there is a special region of pulsational instability for early $\mathrm{F}$ dwarfs which is not at present understood. Current models do not predict pulsational instability of low-degree $g$-modes in these stars. It is therefore unlikely that the $\kappa$ mechanism which is responsible for the $p$-mode instability in $\delta$ Scuti stars will also apply in these stars.

\section{References}

Balona, L.A., Krisciunas, K., \& Cousins, A.W.J. 1994, MNRAS, 270, 905

Krisciunas, K., Griffin, R.F., Guinan, E.F., Luedeke, K.D., \& McCook, G.P. 1995, MNRAS, in press

Mantegazza, L., Poretti, E., \& Zerbi, F.M. 1994, MNRAS, 270, 439 